\title{
High Intensity Light or Pulse Light Sterilization
}

National Cancer Institute

\section{Source}

National Cancer Institute. High Intensity Light or Pulse Light Sterilization. NCI Thesaurus. Code C101693.

A sterilization process that involves the pulsing of a high-power xenon lamp. This process kills microorganisms through the high ultraviolet content of the light and the brief heating effects. 